\title{
Ethanol Extract of Root of Prunus persica Inhibited the Growth of Liver Cancer Cell HepG2 by Inducing Cell Cycle Arrest and Migration Suppression
}

\author{
Hongchun Shen, ${ }^{1}$ Honglian Wang, ${ }^{2}$ Li Wang, ${ }^{2}$ Lu Wang, ${ }^{3}$ Menglian Zhu, ${ }^{4}$ Yao Ming, \\ Sha Zhao, ${ }^{4}$ Junming Fan, ${ }^{5}$ and En Yin Lai ${ }^{6}$ \\ ${ }^{1}$ College of Integrated Chinese and Western Medicine, Southwest Medical University, Luzhou, Sichuan 646000, China \\ ${ }^{2}$ Laboratory of Organ Fibrosis Prophylaxis and Treatment by Combine Traditional Chinese and Western Medicine, \\ Research Center of Combine Traditional Chinese and Western Medicine, Affiliated Traditional Medicine Hospital of \\ Southwest Medical University, Luzhou, Sichuan 646000, China \\ ${ }^{3}$ Department of Nephrology, The Affiliated Hospital of Southwest Medical University, Luzhou, Sichuan 646000, China \\ ${ }^{4}$ Department of Nephrology, The Affiliated Traditional Medicine Hospital of Southwest Medical University, Luzhou, \\ Sichuan 646000, China \\ ${ }^{5}$ Chengdu Medical College, Chengdu, Sichuan 610041, China \\ ${ }^{6}$ Department of Physiology, Zhejiang University School of Medicine, Hangzhou 310000, China
}

Correspondence should be addressed to En Yin Lai; laienyin@zju.edu.cn

Received 14 May 2017; Revised 9 August 2017; Accepted 21 August 2017; Published 12 October 2017

Academic Editor: Raffaele Capasso

Copyright (c) 2017 Hongchun Shen et al. This is an open access article distributed under the Creative Commons Attribution License, which permits unrestricted use, distribution, and reproduction in any medium, provided the original work is properly cited.

\begin{abstract}
Liver cancer is the second most lethal cancer and hepatocellular carcinoma (HCC) is the primary cancer subgroup. However, the current chemotherapy agents remain ineffective and present wide side effects for advanced HCC patient. In this study, we investigated the antitumor role of ethanol extract of root of peach tree (Prunus persica (L.) Batsch and hereafter designated as TSG in short of its Chinese name), which is an important ingredient in Chinese medicine prescription, in liver cancer cell HepG2. By cell viability assay, we showed that addition of TSG in the culture medium inhibited the cell growth of HepG2 cells in a dose and time-dependent way. Cell cycle analysis indicated that TSG caused sustained M/G2 phase arrest. The expression of mitosis-related protein Cdc25c was impaired upon TSG treatment. Furthermore, wound healing assay demonstrated that TSG treatment notably suppressed the migration of HepG2 cells and the expression of extracellular matrix metalloprotease, MMP3 and MMP9. Most significantly, administration of TSG inhibited in vivo tumor growth in nude mice. Our findings suggested that TSG may serve as a source to isolate anti-HCC therapeutic ingredients.
\end{abstract}

\section{Introduction}

According to the epidemiological data from the world health organization, Liver cancer is the second most lethal cancer type around the world. And, hepatocellular carcinoma (HCC) is the most common primary liver cancer [1]. Although early-staged HCC can be subjected to curative surgical treatments, like tumor resection and ablation, the majority of HCC patients often already progress to intermediate or advanced disease stage upon clinical diagnosis. In such case, palliative treatment is the primary medical choice.
However, even the first line palliative treatment medicine, sorafenib, contributes modest survival benefit (about 2.5 months) to advanced HCC patient $[2,3]$. Thus, there is an urgent demand for developing novel therapeutic drugs for future HCC therapy.

Prunus persica (L.) Batsch, peach tree or taoshu in Chinese, originated from Northwest China and nowadays is a worldwide cultivated fruit plant [4]. Beyond usage as a food source, the physical parts of peach tree also showed biological activity and contained constituents with medical effects. Like other fruits, the juicy flesh of peach contains 
various kinds of secondary metabolites with antioxidant and anti-inflammation activity [5]. In addition, physical parts of Prunus persica are used as important ingredients in traditional Chinese medicine prescriptions. Prunus persica has been included in the traditional Chinese medicine prescription to treat blood stasis [6]. Fukuda et al. reported that several isolated glycosides from Prunus persica seeds can inhibit in vitro and in vivo tumorigenesis [7]. Recently, it is shown that the polyphenolic extract of Prunus persica fruit can suppress breast cancer cell proliferation and tumor growth [8, 9]. Furthermore, bark extract of Prunus africana, also named Pygeum africanum and a species showing evolutionarily close relationship with Prunus persica, has been successfully used in the treatment of benign prostatic hyperplasia $[10,11]$.

Root of Prunus persica serves as another important ingredient in traditional Chinese medicine prescriptions. It is described to have the function to treat disease like jaundice, hematemesis, hemorrhoids, amenorrhea, and tinea in classical Chinese medical documents and also used in nowadays Chinese medicine application. The preliminary pharmacological study indicated that root of Prunus persica have anti-inflammation activity [12]. In experience-based clinical application, root of Prunus persica was also applied to treat esophageal cancer [13]. In this study, we firstly investigated the antitumor effect of ethanol extract of root of Prunus persica on the hepatocarcinoma cell HepG2. Our results indicated that the ethanol extract of root of Prunus persica can impair HepG2 cell viability and suppress cell growth through cell cycle arrest and inhibit cell migration. Importantly, administration of ethanol extract of root of Prunus persica can efficiently suppress the tumor growth in nude mice.

\section{Materials and Methods}

2.1. Drug and Cell Line. The dry root of Prunus persica (L.) Batsch, also taoshugen (TSG) in its Chinese name, was subjected to mechanical comminution followed by ethanol extraction for 48 hours at room temperature. The insoluble particles were removed by centrifugation and filtration through a $0.22 \mu \mathrm{m}$ membrane. The bulk ethanol extract was vacuum dried and quantified. For in vitro cellular assay, DMSO was used to redissolve the dried ethanol extract, which can be fully dissolved in DMSO with a maximum solubility of about $0.12 \mathrm{~g} / \mathrm{ml}$. For animal experiment, the dried extract was further redissolved in ethanol and intragastrically administrated to the nude mice at indicated concentration in $10 \%$ ethanol. HepG 2 cell line was a kind gift from Professor Qin Zhou from Chongqing Medical University and was cultured in DMEM supplemented with 10\% FBS (Gemini, USA) at $37^{\circ} \mathrm{C}$ with $100 \%$ humidity and $5 \% \mathrm{CO}_{2}$.

2.2. Cell Viability Assay. Total cell viability was determined with CellTiter96@ AaQueous One Solution (promega, USA). HepG2 cells were seeded on 96-well plate at a density of 3000 cells per well. On the next day, medium containing drugs of indicated concentration or equivalent DMSO was added. 24 hours later, medium was replaced with fresh growth medium containing $10 \%$ of the above cell viability assay reagent and incubated for 1 hour. The plate was analyzed with BioTek synergy 2 microplate reader (USA). All treatments were performed in 4 replicates on the same plate.

2.3. Cell Cycle Analysis. Cell cycle analysis was conducted with the cell cycle detection kit (KeyGen, China) according to the manufacturer's instruction. Briefly, cells subjected to indicated treatment were harvested by regular trypsin digestion and rinsed with PBS. After fixation in 70\% ethanol overnight at $-20^{\circ} \mathrm{C}$, cells were rinsed again in PBS and stained in staining solution containing PI and RNase A. Flow cytometry (Canto II, BD bioscience, USA) was employed to analyze the cellular DNA content. Each treatment was performed in triplicate.

2.4. Western Blotting. For western blotting, $1 \times 10^{6}$ cells were seeded onto $6 \mathrm{~cm}$ dish. After subjecting to indicated treatment for 24 hours, the cells were detached with trypsin digestion and rinsed with PBS followed by lysis with RIPA buffer and centrifugation to remove debris. Protein concentration was determined with the coomassie brilliant blue method. Western blotting was performed as previously described [14]. Antibodies used in this study included rabbit anti-Cdc25c polyclonal antibody (Cat\# D154112, Sangon Biotech, China), rabbit anti-CDK1 polyclonal antibody (Cat\# D160158, Sangon Bio-tech, China), rabbit anti-MMP9 polyclonal antibody (Cat\# 10375-2-AP, Proteintech, USA), rabbit anti-MMP3 polyclonal antibody (Cat\# 17873-1-AP, Proteintech, USA), mouse anti- $\beta$-Actin monoclonal antibody (Cat\# TA-09, ZSGB-Bio, China), HRP-conjugated goat anti-mouse polyclonal antibody (Cat\# ZB2305, ZSGB-Bio, China), and HRP-conjugated goat anti-rabbit polyclonal antibody (Cat\# ZB2301, ZSGB-Bio, China).

2.5. Wound Healing Assay. HepG2 cells were seeded onto 6well plate at a density of $2 \times 10^{6}$ per well. Next day, uniform scratch was made with a pipette tip on the culture surface with $100 \%$ cell confluence. The well was rinsed with fresh blank DMEM medium two times and the cells were maintained in another $2 \mathrm{ml}$ of blank DMEM medium or DMEM medium with $0.34 \mathrm{mg} / \mathrm{ml}$ TSG. Cell migration status was monitored at indicated time point under microscope and represented by the recovery of the scratch.

2.6. In Vivo Animal Experiment. The athymic BALB/c nude mice (BALB/cJNju-Foxnlnu/Nju, 2-month old with body weight of about $17 \mathrm{~g}$, Dashuo Biotech) were subcutaneously inoculated with $1 \times 10^{6}$ HepG 2 cells on both sides of the posterior back, respectively. Since the 2 nd day after cancer cell inoculation, the mice were intragastrically administrated with $4.3 \mathrm{mg} /$ day of TSG or equivalent volume of solvent ( $10 \%$ ethanol) for 4 weeks. Each group contains 10 mice. The concentration of the stock ethanol extract of TSG is $0.086 \mathrm{~g} / \mathrm{ml}$ which almost reaches saturation. In consideration of a maximum administration dosage, the maximum volume tolerance of intragastrical drug administration and avoiding hazards from high concentration of ethanol, the stock TSG ethanol extract was diluted 10 -fold with distilled water (finally in $10 \%$ ethanol) and intragastrically administrated to nude 


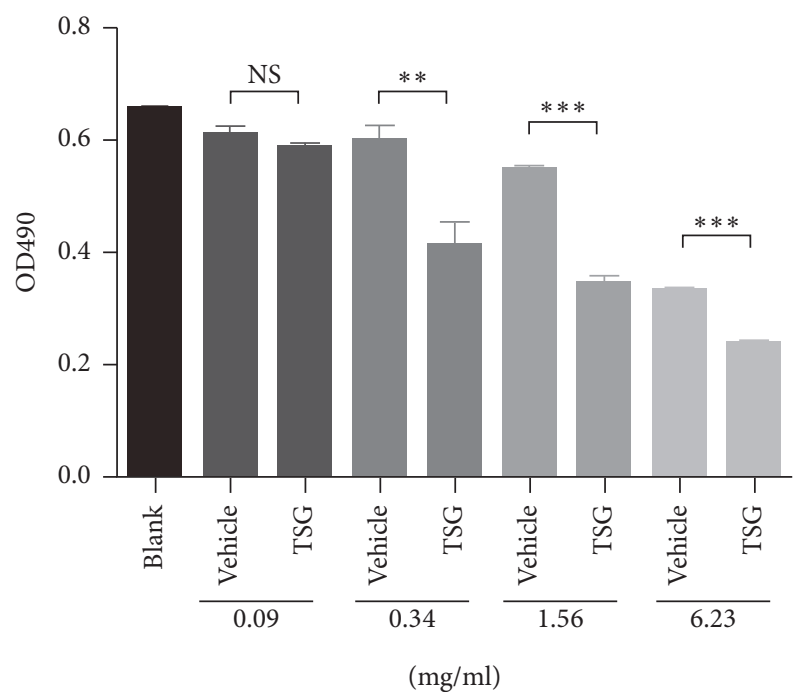

(a)

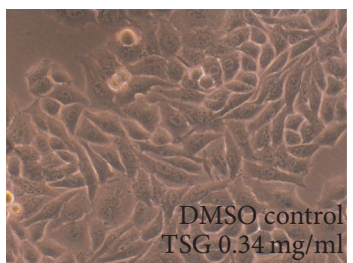

(c)

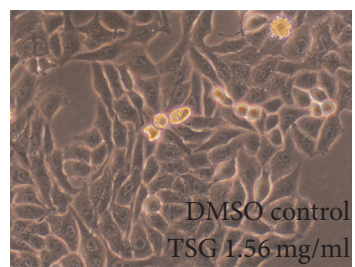

(d)

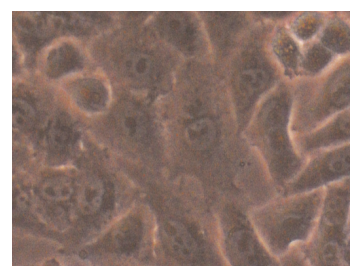

(g)

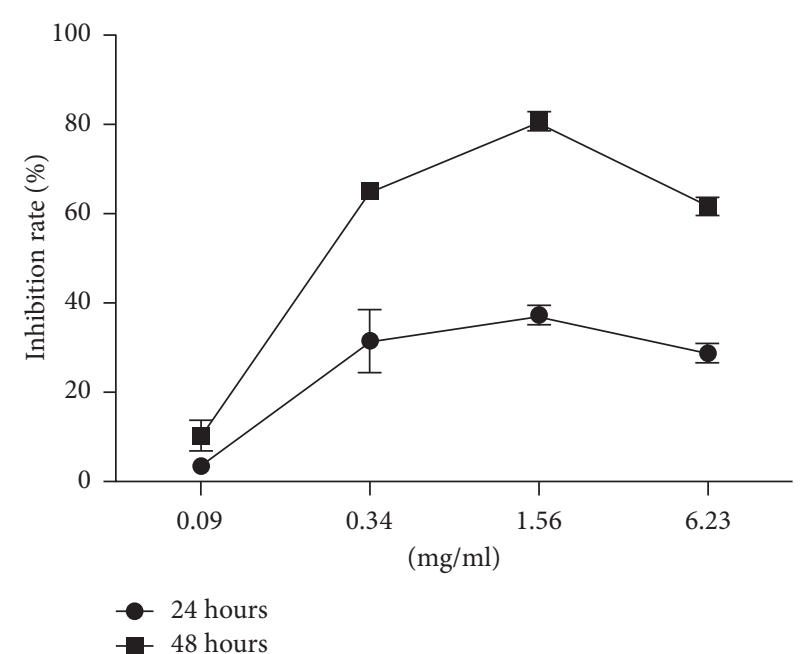

(b)

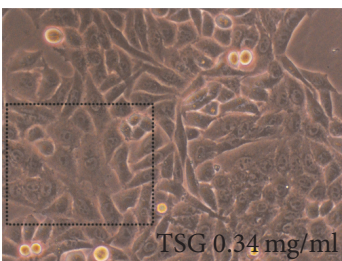

(e)

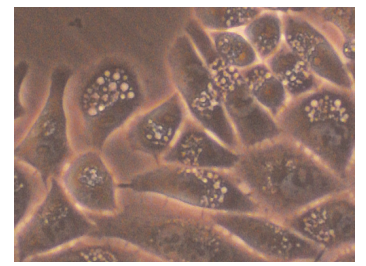

(h)

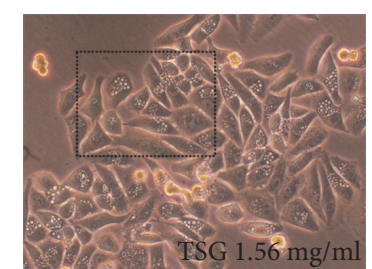

(f)

FIGURE 1: TSG inhibited growth of HepG2 cells. (a) The HepG2 cells were treated with TSG of various concentration for 24 hours followed by determination of total cell viability by viability assay. ${ }^{* *} p<0.01 .{ }^{* * *} p<0.001$. NS: no statistical significance. (b) The dosage and timedependent growth inhibition rate was calculated based on data from viability assay. ((c)-(h)) Morphological change of HepG2 cells treated with TSG and corresponding vehicle control for 24 hours. (g) and (h) are enlarged image in (e) and (f), respectively.

mice in $0.5 \mathrm{ml} /$ day (with a final dosage of $4.3 \mathrm{mg} /$ day). At the end of drug treatment, the mice were anaesthetized by pentobarbital sodium and the tumors were harvested followed by photography and weighing. The animal experiment was approved by the animal ethics committee of Southwest Medical University and conformed to corresponding regulations.

\section{Results}

3.1. TSG Induced Hep G2 Cell Growth Inhibition. To begin the anti-HCC activity investigation of root of Prunus persica, we firstly performed the ethanol extraction of root of Prunus persica (TSG thereafter). For cell experiment, the solvent ethanol was replaced with DMSO as described in the method section. To test the effect of TSG on the growth of liver cancer cell HepG2, we treated the cells with TSG of different concentration and the cell viability was monitored at 24 and 48 hours. The relative growth inhibition rate was calculated with the corresponding vehicle (DMSO) group as control since DMSO were observed to demonstrate slight toxicity on HepG2 cells when used at higher concentration. As shown in Figure 1, compared to vehicle group, TSG treatment induced time and dosage-dependent growth inhibition with a decreased total cell viability. $1.56 \mathrm{mg} / \mathrm{ml}$ TSG treatment for 24 hours can cause 37\% growth inhibition (Figures 1(a) and 1(b)). Morphologically, TSG treatment caused cytoplasmic vacuolization which also demonstrated a dosage effect (Figures $1(\mathrm{c})-1(\mathrm{~h}))$.

3.2. TSG Treatment Caused Cell Cycle Arrest in G2/M Phase. To investigate the factors contributing to the inhibited growth of HepG2 cells, we checked the effect of TSG on cell cycle. As 

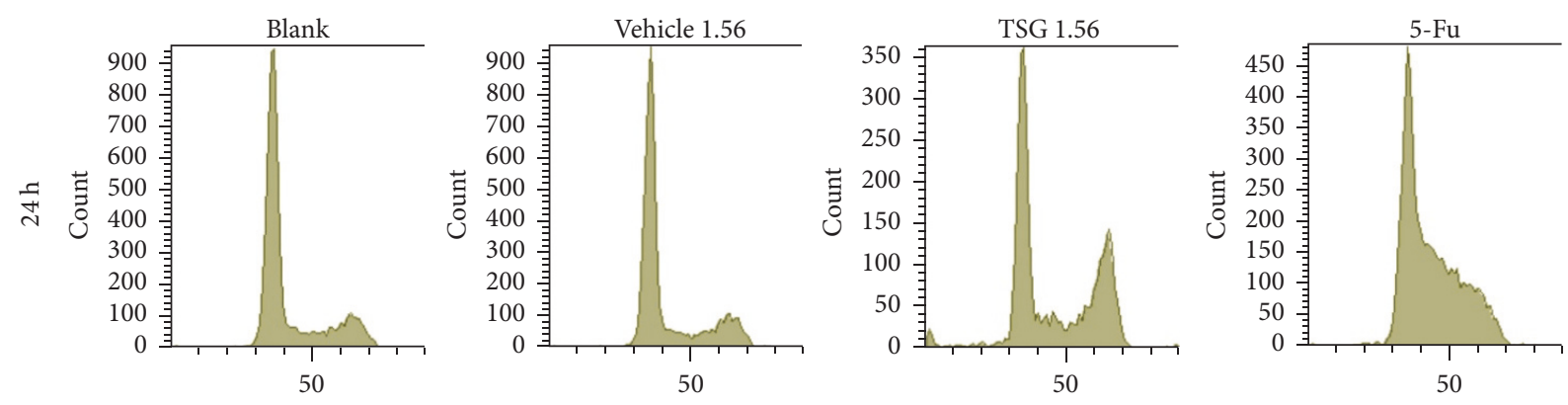

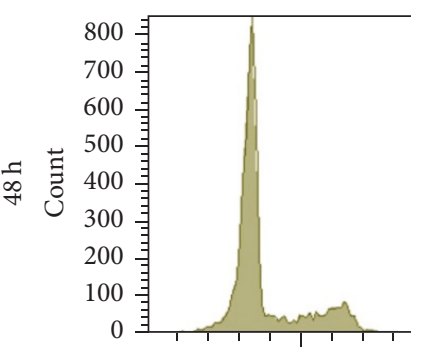

50

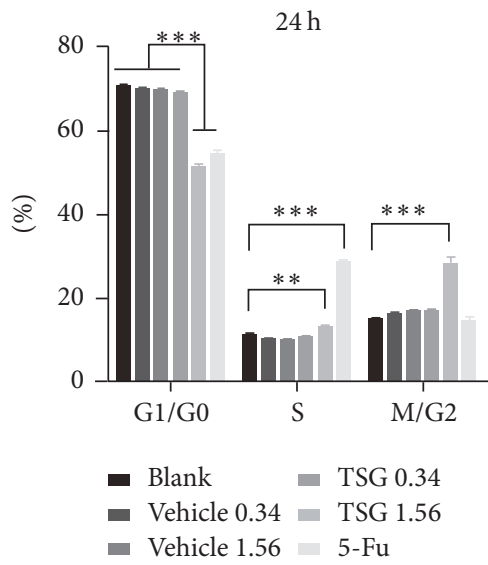

(b)

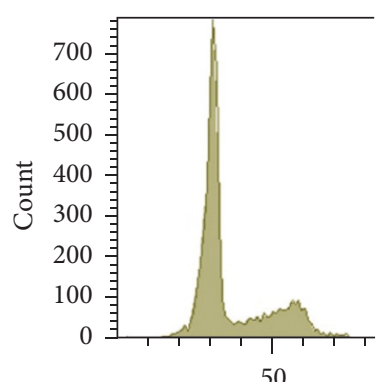

(a)

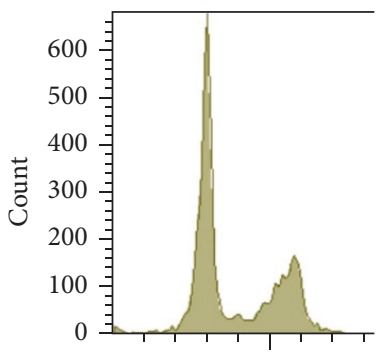

50

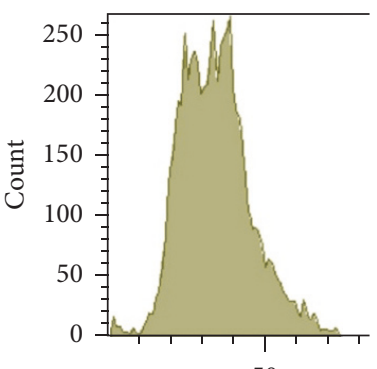

50

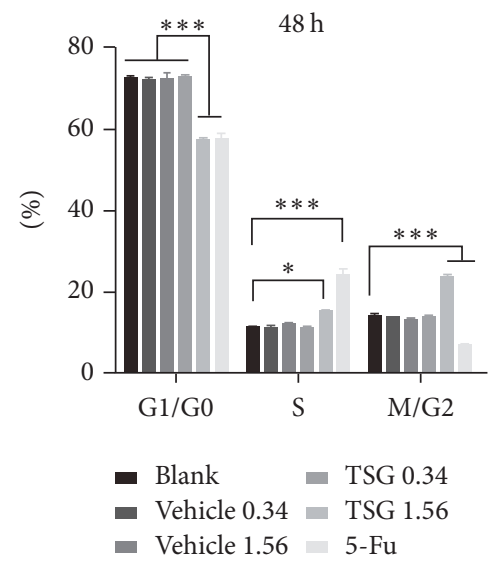

(c)

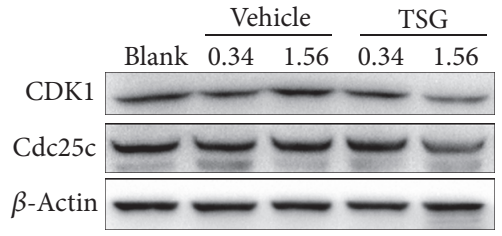

(d)

FIGURE 2: TSG treatment induced G2/M phase arrest of HepG2 cells. (a) Flow cytometry analysis of DNA content of HepG2 cells subjected to indicated treatment. ((b), (c)) Statistical analysis of cell subpopulation ratios for each phase of cell cycle. ${ }^{*} p<0.05 .{ }^{* *} p<0.01 .{ }^{* * *} p<0.001$. (d) Western blotting analysis to detect the expression of CDK1 and Cdc25c.

revealed by PI staining followed by flow cytometry analysis, high-dosage of TSG $(1.56 \mathrm{mg} / \mathrm{ml})$ significantly disturbed the cell cycle with increased M/G2 subgroup and decreased G1/G0 subgroup $(p<0.001)$. And this cell cycle arresting effect can last for at least 2 days. However, treatment with DMSO and low-dosage TSG $(0.34 \mathrm{mg} / \mathrm{ml})$ had no influence on cell cycle (Figures 2(a)-2(c)). As a control, 5-fluorouracil $(5-\mathrm{Fu}, 5 \mu \mathrm{g} / \mathrm{ml})$ also demonstrated vigorous disturbance of HepG2 cell cycle which keeps in line with the previous report [15]. To unravel the underlying molecular event associated with the altered cell cycle pattern, western blotting was used to analyze the key factors regulating cell cycle progress. As shown in Figure 2(d), the protein expression level of Cdc25c and $\mathrm{CDK} 1$, the key proteins modulating mitosis-phase entry, decreased upon treatment with high dose of TSG. In line with the DNA content analysis, low dose of TSG and DMSO (vehicle) showed no influence on protein level of Cdc25c and CKD1. These findings suggested that TSG induced cell cycle arrest at proper concentration.

3.3. The Migration Ability of HepG2 Cells Was Impaired by TSG. As increased migration is another important feature of cancer cell, we then turned to investigate the influence of TSG on migration ability of HepG2 cells. The wound healing assay was employed to analyze the migration ability of HepG2 cells treated with TSG in basal medium without serum. As higher concentration of TSG $(1.56 \mathrm{mg} / \mathrm{ml})$ caused severe cell death in serum-free condition, the lower TSG dose $(0.34 \mathrm{mg} / \mathrm{ml})$ was used in this assay (data not shown). As shown in Figures 3(a) and 3(b), TSG-treated cells demonstrated notable compromised migration since early 6 hours after drug administration. This impaired migration was even more obvious with 


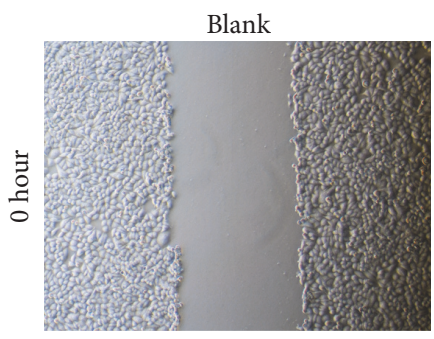

(a)

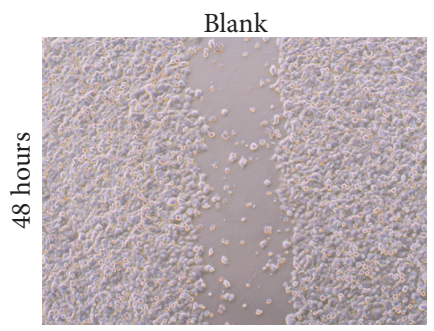

(d)

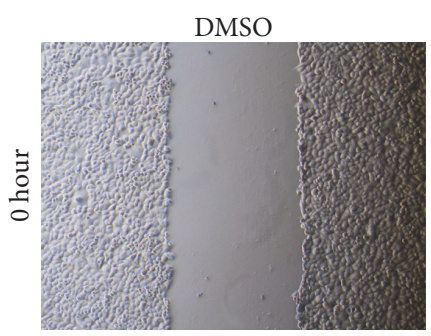

(b)

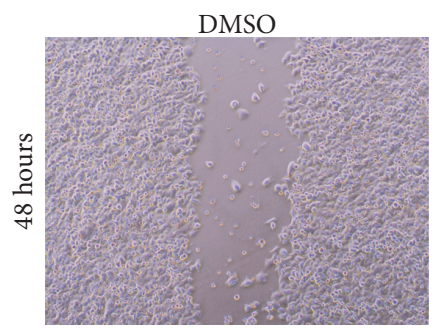

(e)

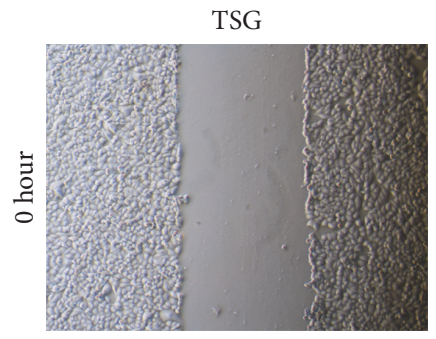

(c)

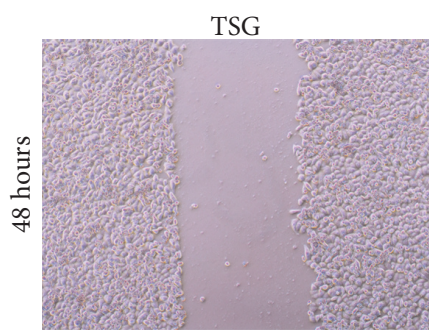

(f)

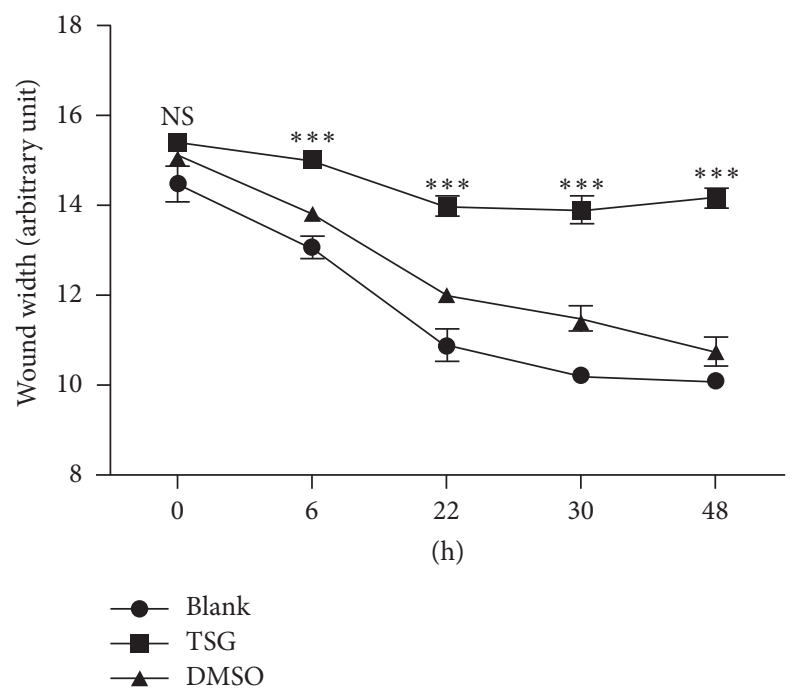

(g)

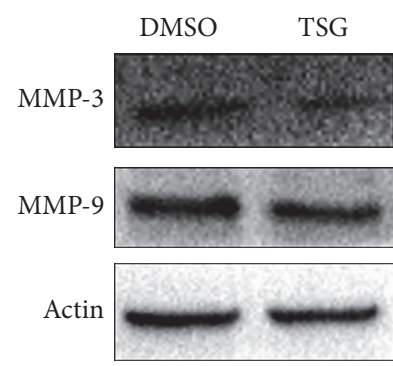

(h)

FIGURE 3: TSG suppressed HepG2 cell migration. ((a)-(f)) Representative images of wound healing assay of HepG2 cells treated with $0.34 \mathrm{mg} / \mathrm{ml}$ TSG or equal volume of DMSO or blank medium at the time point of 0 and 48 hours. (g) Statistical analysis of wound width of HepG2 cells with indicated treatment at indicated time point. ${ }^{* * *} p<0.001$ versus TSG and DMSO group. (h) Western blotting analysis of protein level of MMP-3 and MMP-9.

longer time of TSG treatment. In contrast, the addition of DMSO had no effects on cellular migration compared with the control (blank) (Figures 3(a)-3(g)). Accordingly, TSG treatment attenuated the expression level of the migration marker protein MMP3 and MMP9 as revealed by western blotting (Figure 3(h)).

3.4. TSG Inhibited In Vivo Tumor Growth in Nude Mice. To unravel whether TSG can present a suppression activity on in vivo tumor growth, we established the live tumor model by subcutaneously transplanting HepG2 cells in nude mice which were subjected to intragastrical administration of
TSG ( $4.3 \mathrm{mg} / \mathrm{day})$ or vehicle solution. One month later, the TSG-treated nude mice developed smaller tumors in respect to both size and weight compared with the vehicle group (Figures 4(a) and 4(b)). These results implicated that TSG can partially inhibit in vivo tumor growth derived from HepG2 cells.

\section{Discussion}

Prunus persica is an important source of medicinal materials in traditional Chinese medicine. Physical parts of Prunus persica, like flower, seed, and gum, have been used to cure 


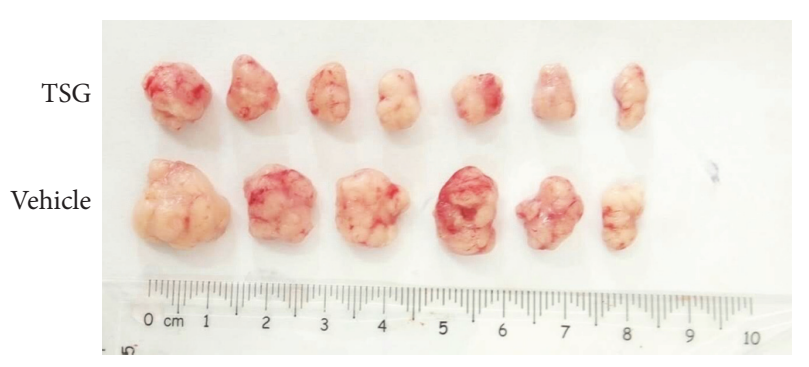

(a)

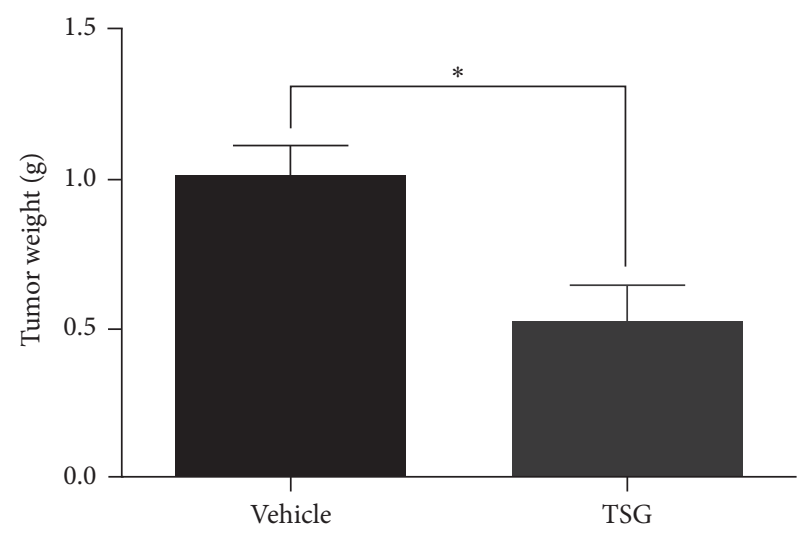

(b)

FIGURE 4: TSG attenuated HepG2-derived in vivo tumor growth. (a) Representative image of isolated tumors from tumor-bearing nude mice treated with TSG or solvent solution. (b) Statistical analysis of weight of isolated tumors. ${ }^{*} p<0.05$ versus control group.

disease for hundreds of years. Root of Prunus persica is applied to treat jaundice, hematemesis, hemorrhoids, amenorrhea, and tinea in Chinese medicine. In experience-based folk medicine, root of Prunus persica is also included in the prescription to ameliorate esophageal cancer [13]. As far as we know, only one study described the toxicity of root of Prunus persica on liver cancer cell lines SMMC7721 and SKHEP-1 [13]. However, no detailed mechanism on its antitumor effect was provided. In this study, we presented the evidence that ethanol extract of root of Prunus persica can inhibit the growth of HepG2 cells in vitro by impairing cell cycle and migration. Most importantly, ethanol extract of root of Prunus persica can slow down the in vivo growth of HepG2 cells-derived tumor in nude mice.

Uncontrolled cell proliferation, apoptosis, cell cycle, and enhanced cell migration are the key features of tumor cells and also the important therapeutic targets. In line with the previous report in liver cancer cell SMMC7721 and SK-HEP1, our data indicated that root of Prunus persica extract also induced a dose and time-dependent growth inhibition of HepG2 cells. Furthermore, cell cycle analysis demonstrated a sustained M/G2 phase arrest. However, we found that the apoptosis of HepG2 cells showed little change upon treatment with root of Prunus persica extract at the concentration tested (data not shown). On the other hand, migration of HepG2 cells was significantly impeded by root of Prunus persica extract companioned with decreased expression of extracellular matrix metalloprotease, MMP3 and MMP9. These data implied that root of Prunus persica extract-induced HepG2 growth inhibition may be attributed to cell cycle arrest and migration suppression.

Furthermore, it is recently reported that the polyphenolic extract of Prunus persica fruit can inhibit the proliferation of in vitro breast cancer cell and in vivo breast tumor growth and lung metastasis $[8,9,16]$. The Prunus persica fruit and its polyphenolic extract can also execute chemoprotective role against cancer through modulating in vivo xenobiotic metabolizing enzymes [17]. Fukuda et al. reported that
Prunus persica seed-derived glycosides showed antitumor activity in vitro and in vivo [7]. Combining these studies with our findings, we may speculate that many physical parts of Prunus persica tree contain antitumor components. However, more studies are needed to confirm this hypothesis.

By now, chemotherapeutic palliative treatment is almost the only choice for advanced liver HCC. However, molecular targeting agents, like multitarget kinase inhibitor sorafenib and sunitinib, contribute limited survival benefit to HCC patients $[3,18]$. And cytotoxic agents, like cisplatin and doxorubicin, show almost no therapeutic response [3]. Most importantly, both of the molecular targeting agents and cytotoxic agents demonstrate severe side effects [3]. On the contrary, root of Prunus persica is a natural material and used as medical ingredient for hundreds of years in Chinese medicine. The long time clinical application suggests its relative safety. Thus, it should be a good source to explore novel HCC chemotherapy agent. However, strictly controlled study is needed to assess the safe dosage and potential side effects before its translation to clinical application. On the other hand, we should note that the ethanol extract of root of Prunus persica used in this study is a complex mixture may containing several different therapy-effective components and also impurities. This makes deep exploration of the tumor suppression role of root of Prunus persica difficult. Thus, our next work is to isolate and identify these medical active ingredients to further clarify their anti-HCC mechanism.

\section{Conclusion}

Ethanol extract of root of Prunus persica can inhibit in vitro HepG2 cells growth by inducing cell cycle arrest and migration suppression and in vivo tumor growth, suggesting potential application for novel anti-HCC drug development.

\section{Conflicts of Interest}

The authors declared no conflicts of interest. 


\section{Authors' Contributions}

Hongchun Shen and Honglian Wang contributed equally to this work.

\section{Acknowledgments}

This research was supported by China Postdoctoral Science Foundation (2014M552544XB), General Project of Education Department in Sichuan (13ZB0256), and Joint Project of Luzhou Science and Technology Department and Southwest Medical University (2013LZLY-J34).

\section{References}

[1] W. H. Organization, Estimated Cancer Incidence, Mortality and Prevalence Worldwide in 2012, International Agency for Research on Cancer, 2012.

[2] J. Bruix and M. Sherman, "Management of hepatocellular carcinoma: an update," Hepatology, vol. 53, no. 3, pp. 1020-1022, 2011.

[3] M. Omata, L. A. Lesmana, R. Tateishi et al., "Asian pacific association for the study of the liver consensus recommendations on hepatocellular carcinoma," Hepatology International, vol. 4, no. 2, pp. 439-474, 2010.

[4] M. Faust and B. Timon, "Origin and dissemination of peach," Horticultural Reviews, vol. 17, p. 331, 2010.

[5] J. Gasparotto, N. Somensi, R. C. Bortolin et al., "Effects of different products of peach (Prunus persica L. Batsch) from a variety developed in southern Brazil on oxidative stress and inflammatory parameters in vitro and ex vivo," Journal of Clinical Biochemistry and Nutrition, vol. 55, no. 2, pp. 110-119, 2014.

[6] D. H. Kim, S. M. Lee, Y. J. Lee et al., "Effect of Paeotang on tumor necrosis factor $\alpha$-induced vascular inflammation in human umbilical vein endothelial cells," Chinese Journal of Integrative Medicine, pp. 1-10, 2017.

[7] T. Fukuda, H. Ito, T. Mukainaka, H. Tokuda, H. Nishino, and T. Yoshida, "Anti-tumor promoting effect of Glycosides from Prunus persica seeds," Biological \& Pharmaceutical Bulletin, vol. 26, no. 2, pp. 271-273, 2003.

[8] G. Noratto, W. Porter, D. Byrne, and L. Cisneros-Zevallos, "Polyphenolics from peach (Prunus persica var. Rich Lady) inhibit tumor growth and metastasis of MDA-MB-435 breast cancer cells in vivo," The Journal of Nutritional Biochemistry, vol. 25, no. 7, pp. 796-800, 2014.

[9] M. Vizzotto, W. Porter, D. Byrne, and L. Cisneros-Zevallos, "Polyphenols of selected peach and plum genotypes reduce cell viability and inhibit proliferation of breast cancer cells while not affecting normal cells," Food Chemistry, vol. 164, pp. 363-370, 2014.

[10] J. Breza, O. Dzurny, A. Borowka et al., "Efficacy and acceptability of Tadenan ${ }^{\circledR}$ (Pygeum africanum extract) in the treatment of benign prostatic hyperplasia (BPH): A multicentre trial in central Europe," Current Medical Research and Opinion, vol. 14, no. 3, pp. 127-139, 1998.

[11] S. Larré, P. Camparo, E. Comperat et al., "Biological effect of human serum collected before and after oral intake of Pygeum africanum on various benign prostate cell cultures," Asian Journal of Andrology, vol. 14, no. 3, pp. 499-504, 2012.

[12] J. Xia and L. Huagang, "Experimental study of anti-inflammation activity of root of prunus persica," Journal of Chinese Medicinal Materials, vol. 29, no. 8, pp. 821-823, 2006 (Chinese).
[13] Z. Jingquan, H. Deqiang, Z. Kunhe et al., "Experimental study of cytotoxicity on hepatocarcinoma with root of wild peach tree," Acta Academiae Medicinae Jiangxi, vol. 41, no. 4, pp. 17-19, 2001 (Chinese).

[14] N. Mao, R.-Z. Tan, S.-Q. Wang et al., "Ginsenoside Rg1 inhibits angiotensin II-induced podocyte autophagy via AMPK/mTOR/PI3K pathway," Cell Biology International, vol. 40, no. 8, pp. 917-925, 2016.

[15] Z. Zhang, L. Miao, C. Lv et al., "Wentilactone B induces G2/M phase arrest and apoptosis via the Ras/Raf/MAPK signaling pathway in human hepatoma SMMC-7721 cells," Cell Death \& Disease, vol. 4, no. 6, article no. e657, 2013.

[16] G. Noratto, W. Porter, D. Byrne, and L. Cisneros-Zevallos, "Identifying peach and plum polyphenols with chemopreventive potential against estrogen-independent breast cancer cells," Journal of Agricultural and Food Chemistry, vol. 57, no. 12, pp. 5219-5226, 2009.

[17] D. Canistro, F. Vivarelli, S. Cirillo, G. Costa, C. Andreotti, and M. Paolini, "Comparison between in toto peach (Prunus persica L. Batsch) supplementation and its polyphenolic extract on rat liver xenobiotic metabolizing enzymes," Food and Chemical Toxicology, vol. 97, pp. 385-394, 2016.

[18] P. Intaraprasong, S. Siramolpiwat, and R.-K. Vilaichone, "Advances in management of hepatocellular carcinoma," Asian Pacific Journal of Cancer Prevention, vol. 17, no. 8, pp. 3697-3703, 2016. 


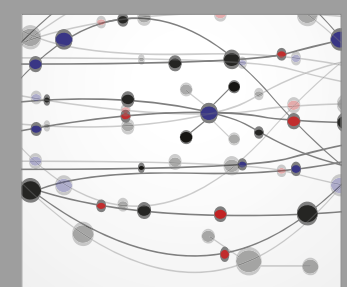

The Scientific World Journal
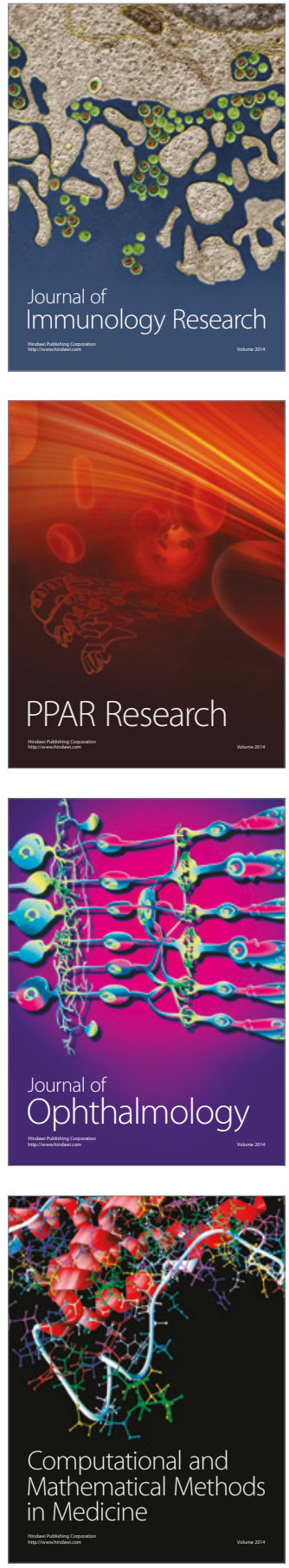

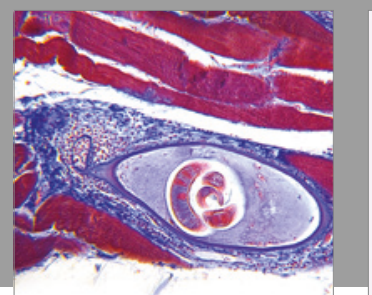

Gastroenterology Research and Practice
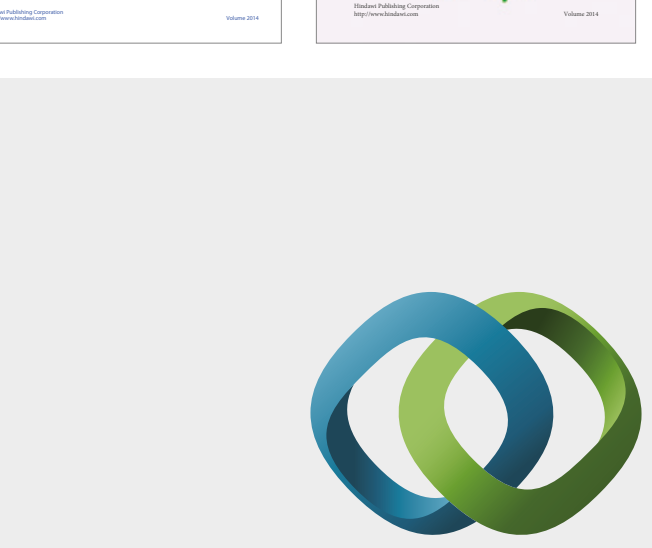

\section{Hindawi}

Submit your manuscripts at

https://www.hindawi.com
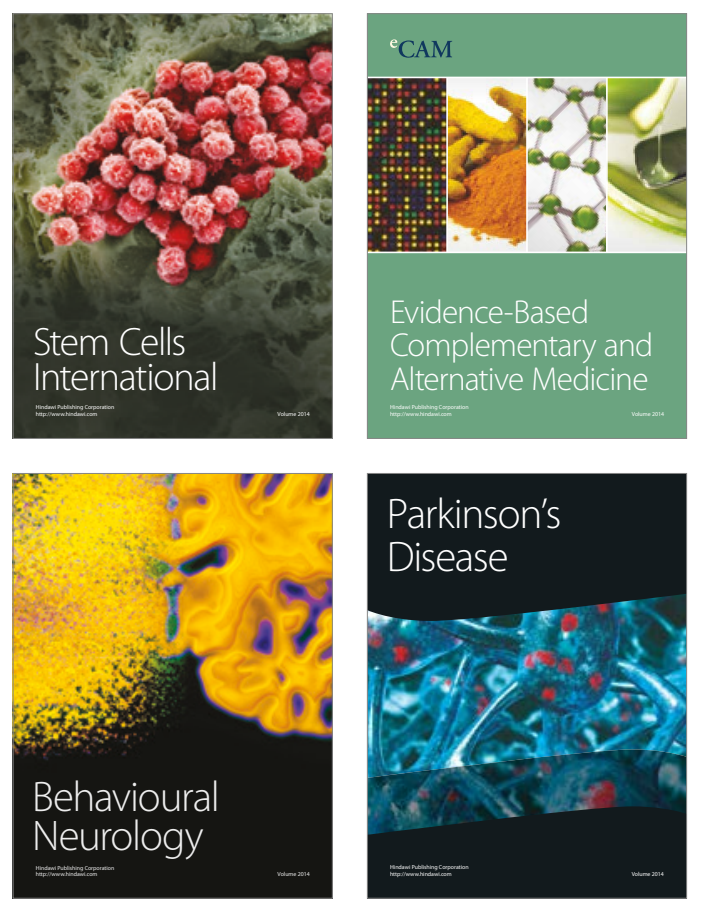
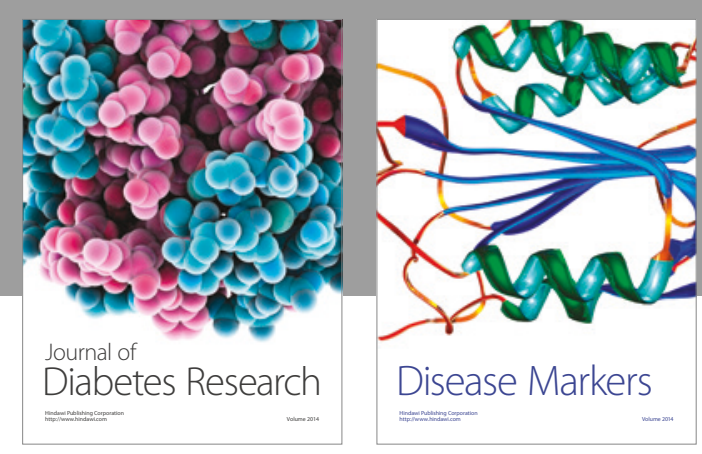

Disease Markers
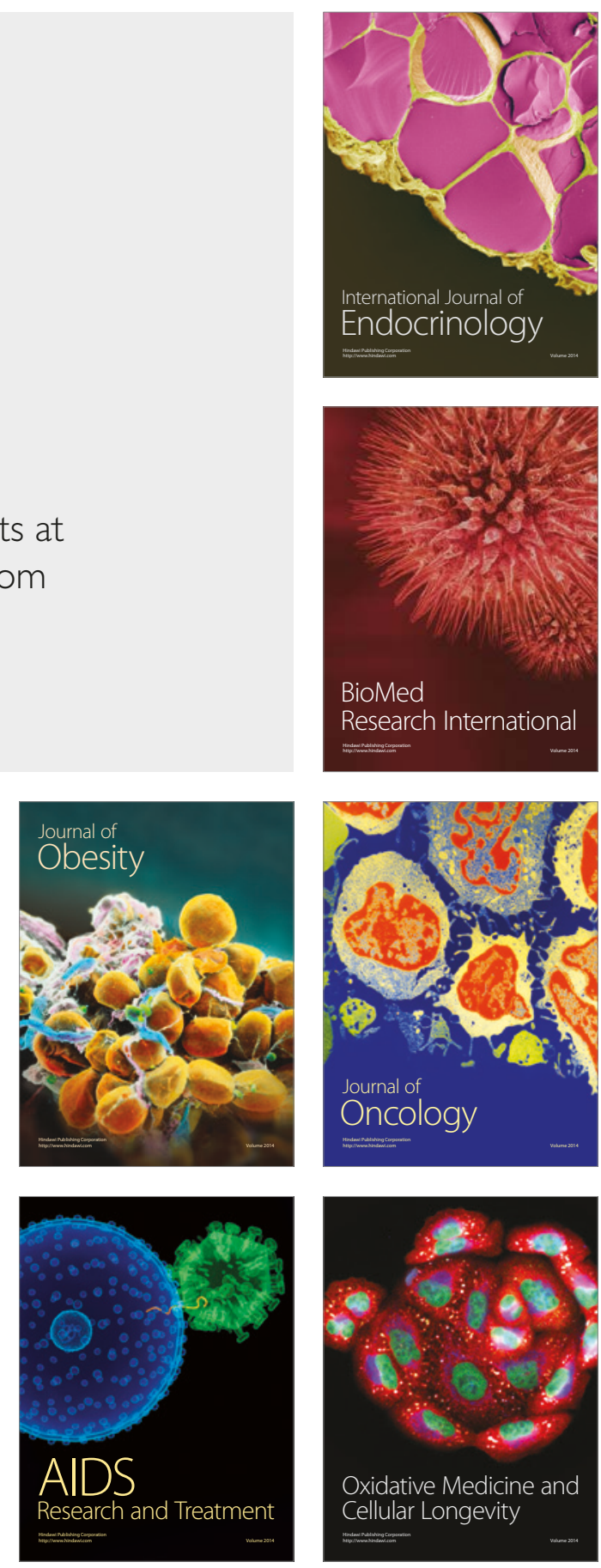